\title{
Enabling time-resolved 2D spatial-coherence measurements using the Fourier-analysis method with an integrated curved-grating beam monitor
}

\author{
Kai Bagschik, ${ }^{1, *}$ Michael Schneider, ${ }^{2}$ Jochen Wagner, ${ }^{3}$ Ralph Buss,,${ }^{3,4}$ \\ Matthias Riepp, ${ }^{1}$ Andre Philippi-Kobs, ${ }^{1}$ (i) Leonard Müller, ${ }^{1,5}$ (i) Wojciech Roseker, ${ }^{1}$ (i) \\ Florian Trinter, ${ }^{1,6}$ Moritz Hoesch, ${ }^{1}$ Jens Viefhaus, ${ }^{7}$ (i) Stefan Eisebitt, ${ }^{2}$ \\ Gerhard Grübel, ${ }^{1,4}$ Hans Peter Oepen, ${ }^{3,4}$ and Robert Frömter ${ }^{3,4}$ (1) \\ ${ }^{1}$ Deutsches Elektronen-Synchrotron (DESY), Notkestraße 85, 22607 Hamburg, Germany \\ ${ }^{2}$ Max-Born-Institut für Nichtlineare Optik und Kurzzeitspektroskopie, Max-Born-Straße 2a, 12489 Berlin, Germany \\ ${ }^{3}$ Institut für Nanostruktur- und Festkörperphysik, Universität Hamburg, CHyN, Luruper Chaussee 149, 22761 Hamburg, Germany \\ ${ }^{4}$ The Hamburg Centre for Ultrafast Imaging, Luruper Chaussee 149, 22761 Hamburg, Germany \\ ${ }^{5}$ Department of Physics, Universität Hamburg, Luruper Chaussee 149, 22761 Hamburg, Germany \\ ${ }^{6}$ Molecular Physics, Fritz-Haber-Institut der Max-Planck-Gesellschaft, Faradayweg 4, 14195 Berlin, Germany \\ ${ }^{7} \mathrm{Helmholtz-Zentrum} \mathrm{Berlin} \mathrm{für} \mathrm{Materialien} \mathrm{und} \mathrm{Energie} \mathrm{GmbH,} \mathrm{Albert-Einstein-Straße} \mathrm{15,} 12489$ Berlin, Germany \\ ${ }^{*}$ Corresponding author: kai.bagschik@desy.de
}

Received 8 July 2020; revised 4 September 2020; accepted 4 September 2020; posted 8 September 2020 (Doc. ID 402264); published 30 September 2020

\begin{abstract}
Direct 2D spatial-coherence measurements are increasingly gaining importance at synchrotron beamlines, especially due to present and future upgrades of synchrotron facilities to diffraction-limited storage rings. We present a method to determine the 2D spatial coherence of synchrotron radiation in a direct and particularly simple way by using the Fourier-analysis method in conjunction with curved gratings. Direct photon-beam monitoring provided by a curved grating circumvents the otherwise necessary separate determination of the illuminating intensity distribution required for the Fourier-analysis method. Hence, combining these two methods allows for time-resolved spatial-coherence measurements. As a consequence, spatial-coherence degradation effects caused by beamline optics vibrations, which is one of the key issues of state-of-the-art X-ray imaging and scattering beamlines, can be identified and analyzed. () 2020 Optical Society of America
\end{abstract}

https://doi.org/10.1364/OL.402264

Realizing the full potential of low-emittance synchrotron facilities in terms of high coherent flux and diffraction-limited focusing at beamlines imposes high standards on X-ray optics design, stability, and quality [1,2]. The big challenge is to preserve the high degree of spatial coherence of the X-ray beam from the undulator source all the way to the user experiment. Tools for beam-coherence diagnostics will play a key role in this context, as the knowledge of the spatial-coherence properties is of vital importance for the preparation and operation of coherence-based experiments, such as coherent diffractive imaging (CDI) [3], Fourier-transform holography (FTH) $[4,5]$, and X-ray photon correlation spectroscopy (XPCS) [6].
Several advanced coherence-measurement techniques have been developed to determine the two-dimensional spatialcoherence properties of synchrotron radiation. Among these techniques are the near-field speckle analysis [7], circular grating interferometry [8], and the Fourier-analysis method [9].

In this Letter, we present the Fourier-analysis method with an integrated curved-grating beam monitor, which allows for a direct and time-resolved 2D spatial-coherence determination. Time-resolved means, in this context, that the 2D spatial-coherence properties can be studied, e.g., at timescales comparable to beamline optics vibrations (ms). As a consequence, spatial-coherence degradation effects caused by optics vibrations can be uncovered and investigated. In our previous studies $[9,10]$, the beam-intensity distribution required for the Fourier-analysis method had to be determined separately by scanning a $1 \mu \mathrm{m}$ pinhole in front of a photodiode, which makes time-resolved spatial-coherence measurements feasible only under certain conditions. Recently, it has been demonstrated that a sample carrying a grating that forms an off-center zoneplate segment can be used for photon-beam monitoring in X-ray transmission experiments, allowing the detection of the photonbeam position as well as its $2 \mathrm{D}$ intensity distribution $[11,12]$. Both the speckle pattern used for the Fourier-analysis method and the photon-beam intensity distribution are recorded simultaneously on the CCD detector. Hence, the Fourier-analysis method along with curved gratings circumvents the additional determination of the required beam-intensity distribution, and time-resolved spatial-coherence measurements become feasible. As an additional benefit, the presented method can be easily integrated in standard X-ray scattering and imaging setups for 
a quick optimization of the spatial-coherence properties of the illuminating beam prior to or during the experiments.

The experiments reported here were carried out at the XUV variable polarization beamline P04 at PETRA III [13]. The beamline is equipped with an APPLE-II-type helical undulator delivering photon energies in the range of 250-3000 eV. The beam is first deflected in the horizontal direction by a switching mirror unit $35 \mathrm{~m}$ downstream of the undulator and subsequently monochromatized by a monochromator unit $(46 \mathrm{~m})$ consisting of a vertical plane mirror and a varied-linespacing (VLS) grating. The VLS grating focuses the beam in the vertical direction onto the exit-slit unit $(71 \mathrm{~m})$ defining the energy resolution. For the experiments, a $30 \mu \mathrm{m}$ exit-slit size is used, resulting in a resolving power of $10^{4}$ corresponding to a longitudinal coherence length of $l_{c}=10-20 \mu \mathrm{m}$, sufficient for scattering experiments and the Fourier-analysis method. The beam is focused to the experiment in the horizontal and vertical directions using a horizontal $(78.5 \mathrm{~m})$ and vertical $(79.1 \mathrm{~m})$ plane elliptical mirror pair in Kirkpatrick-Baez geometry. The focal spot size at the sample position is $10 \times 10 \mu \mathrm{m}^{2}$.

The holographic imaging (XHM) and small-angle X-ray scattering (SAXS) setup [9], specifically designed for experiments at the P04 beamline, has been used for the spatial-coherence measurements. The sample membrane can be positioned with respect to the beam using a piezoelectrically driven positioning system. The SAXS patterns are recorded by a $16 \mathrm{Mpx}$ CCD camera with a pixel size of $15 \times 15 \mu^{2}$, located $90 \mathrm{~cm}$ downstream of the sample. A $1 \mathrm{~mm}$ diameter beam stop protects the CCD camera from the high-intensity direct beam.

The sample is a spatially disordered nanodot array fabricated out of a homogeneous metallic multilayer $\left(\mathrm{Co}_{1.64 \mathrm{~nm}} / \mathrm{Pt}_{2 \mathrm{~nm}}\right)_{2}$ via nanosphere lithography utilizing di-block copolymer micelles [14]. The multilayer is deposited on the front side of a $200 \mathrm{~nm}$ thick $\mathrm{Si}_{3} \mathrm{~N}_{4}$ membrane of $500 \times 500 \mu \mathrm{m}^{2}$ size. The nanodots have a diameter of $\approx 20 \mathrm{~nm}$ set by the core size, and their distance distribution peaks at $110 \mathrm{~nm}$, set by the shell diameter. A $5 \mathrm{~nm}$ tantalum layer is deposited onto the back side of the $\mathrm{Si}_{3} \mathrm{~N}_{4}$ membrane using DC magnetron sputtering.

The curved grating is milled into the tantalum layer with a nominal dose of $550 \mu \mathrm{C} / \mathrm{cm}^{2}$ using a focused ion beam $\left(\mathrm{Ga}^{+}\right.$ ions, $30 \mathrm{kV}$ acceleration voltage) [11]. For the milling process, the grating with a total area of $25 \times 25 \mu^{2}$ is generated on a grid of $3500 \times 3500$ points with a periodicity varying from approx. $270 \mathrm{~nm}$ to $1900 \mathrm{~nm}$. With the given detector distance, this grating maps a 90-fold magnified image of the entire photon-beam profile incident in the sample plane to two centro-symmetric areas in Fourier space $( \pm 1$ st diffraction orders of the grating). These are centered at a distance of $3 \mathrm{~mm}$ away from the zeroth diffraction order, in both horizontal and vertical directions. Note that we slightly modify the grating formula given in Ref. [12] to yield segments of hyperbolic, rather than Fresnel-type, zone plates. This is achieved by globally shifting all grating orientation angles $\varphi$ by $\pi / 2$, i.e., by substituting $\varphi \rightarrow \varphi+\pi / 2$ in Eq. (1) of Ref. [12]. Compared to Fresnel-type gratings, the hyperbolic variant yields significantly better resolution with respect to sharp intensity gradients in horizontal and vertical directions, while having equivalent resolving power in all other directions. They are, however, more prone to distortions when far-field conditions are not met and produce an image of the illumination function that is rotated by $90^{\circ}$ and mirrored (see Fig. 1).
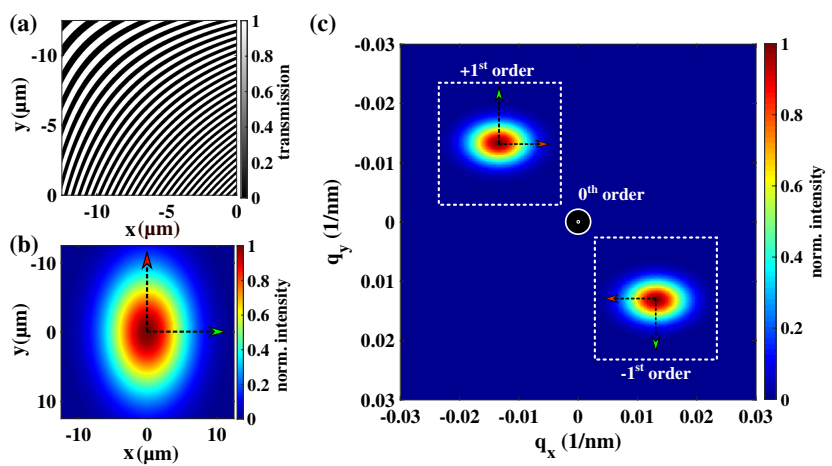

Fig. 1. (a) Binary milling pattern for the hyperbolically curved grating used for the experiments. Only the upper left corner of the $25 \times 25 \mu^{2}$ area is shown. (b) Gaussian photon-beam profile assumed for the calculation $\left(\sigma_{\mathrm{x}, \mathrm{y}}=4 \mu \mathrm{m}\right.$ and $\left.6 \mu \mathrm{m}\right)$. (c) Modeled scattering signature of the curved grating (magnified image of the illuminating photon-beam profile at the +1 st and -1 st diffraction order). The white-dashed rectangles mark the magnified curved-grating areas.

Figure 1(a) shows the upper left corner of the binary milling pattern for the curved grating used in the experiments. The scattering signature of the curved grating in Fourier space can be modeled by means of the Fourier transform of the binary curved-grating pattern [see Fig. 1(c)]. The binary pattern is multiplied with the photon-beam profile, assumed to be Gaussian $\left[4 \times 6 \mu^{2}\right.$ (rms)] as shown in Fig. 1(b), in order to simulate the imaging characteristics of the curved grating. In Fig. 1(c), the +1 st and -1 st diffraction orders of the curved grating show the magnified photon-beam profile. The magnified curved-grating area is marked with a white-dashed rectangle. The zeroth order in the center is blocked by the beam stop in the experiments, and the diffraction pattern of the nanodots is located outside of the shown Fourier space range.

Partially spatial coherent X-ray illumination on the disordered nanodot array with electron density $\rho(r)$ results in a far-field intensity distribution on the CCD detector given by [15]

$$
I(\mathbf{q})=\iint J\left(\mathbf{r}_{1}, \mathbf{r}_{2}\right) \rho\left(\mathbf{r}_{1}\right) \rho\left(\mathbf{r}_{2}\right) \mathbf{e}^{-\mathbf{q}\left(\mathbf{r}_{1}-\mathbf{r}_{2}\right)} \mathrm{d} \mathbf{r}_{1} \mathrm{~d} \mathbf{r}_{2},
$$

where $\mathbf{r}=(x, y)$ is a $2 \mathrm{D}$ vector in the sample plane, $\mathbf{q}$ is a $2 \mathrm{D}$ scattering vector with $|\mathbf{q}|=4 \pi \sin (\theta) / \lambda, \lambda$ is the wavelength, and $2 \cdot \theta$ is the scattering angle. $J\left(\mathbf{r}_{1}, \mathbf{r}_{2}\right)$ is the mutual optical intensity (MOI) and describes the spatial coherence of the illuminating field in the quasi-monochromatic approximation. It can be written, within the Gaussian Schell model (GSM) [16], as

$$
J\left(\mathbf{r}_{1}, \mathbf{r}_{2}\right)=\sqrt{I\left(\mathbf{r}_{1}\right)} \sqrt{I\left(\mathbf{r}_{2}\right)} \mu\left(\mathbf{r}_{1}-\mathbf{r}_{2}\right),
$$

where $\mu\left(\mathbf{r}_{1}-\mathbf{r}_{2}\right)$ is the complex degree of coherence (CDC), and $I\left(\mathbf{r}_{1,2}\right)$ are the intensity values of the illuminating beam at positions $\mathbf{r}_{1,2}$. Both the CDC and intensity $I(\mathbf{r})$ are Gaussian. The rms width of the CDC gives the transverse coherence length $l_{s}$. The Fourier-analysis method provides the full 2D spatialcoherence properties and hence the transverse coherence lengths of the illuminating beam in all angular directions. It is based on the analysis of the inverse Fourier transform of the far-field intensity distribution, given in Eq. (1), expressed by $[9,10]$ 


$$
I_{\mathrm{F}}(\Delta \mathbf{r})=|\mathbf{P}(\Delta \mathbf{r})||\Psi(\Delta \mathbf{r})||\mu(\Delta \mathbf{r})|,
$$

where $\Delta \mathbf{r}=\mathbf{r}_{1}-\mathbf{r}_{2}, \mathbf{P}(\Delta \mathbf{r})$ is the Patterson function of $\rho(r)$, and $\Psi(\Delta \mathbf{r})$ is the autocorrelation function of the illuminating beam-intensity distribution $I(\mathbf{r}) . \mathbf{P}(\Delta \mathbf{r})$ is a constant in the case of the disordered nanodot array, except in the vicinity of the center, and contributes to $I_{\mathrm{F}}(\Delta \mathbf{r})$ only by a constant multiplicative factor. In order to determine the CDC using Eq. (3), and hence $l_{s}$ in $2 \mathrm{D}$, the knowledge of $I(\mathbf{r})$ is required.

In our experiment, the Fourier-analysis method combined with a hyperbolically curved grating is used for a direct $2 \mathrm{D}$ spatial-coherence determination. A SAXS pattern from the nanodot sample and the photon-beam profile imaged via the curved grating are recorded simultaneously in a single exposure $(500 \mathrm{~ms})$ of the CCD detector at a photon energy of $500 \mathrm{eV}$. To image the whole beam, the sample was aligned such that the beam was centered on the grating. Figure 2 shows the elliptical ring-shaped speckle pattern of the sample and the scattering signature of the curved grating close to the center of the CCD detector. In the left half of Fig. 2(a), the central part is masked out, and it shows the speckle signature of the SAXS pattern at higher contrast. For the Fourier-analysis method, the entire center of the SAXS pattern is masked out. The right half shows the scattering signature of the curved grating, and hence the magnified illuminating photon-beam profile, as demonstrated above. The curved grating has been specifically designed so that the photon-beam profile appears and fits into the central region of the detector. In this case, the photon-beam profile is not distorted by the SAXS pattern. Additionally, the groove depth of the curved grating has been tailored such that the diffraction signal fits to the dynamical range of the detector and such that the image of the photon-beam profile and the scattering pattern of the sample both can be detected with sufficient intensity simultaneously. Figure 2(b) shows the 2D photon-beam profile in real-space coordinates (sample plane), which is well described by a 2D Gaussian function. Due to the fact that both the photon-beam profile and the SAXS pattern are recorded in the same image, no additional photon-beam characterization is required. As a consequence, the $2 \mathrm{D}$ spatial-coherence properties of the illuminating beam and hence $\mu(\Delta \mathbf{r})$ can be deduced directly and in a particularly easy way.

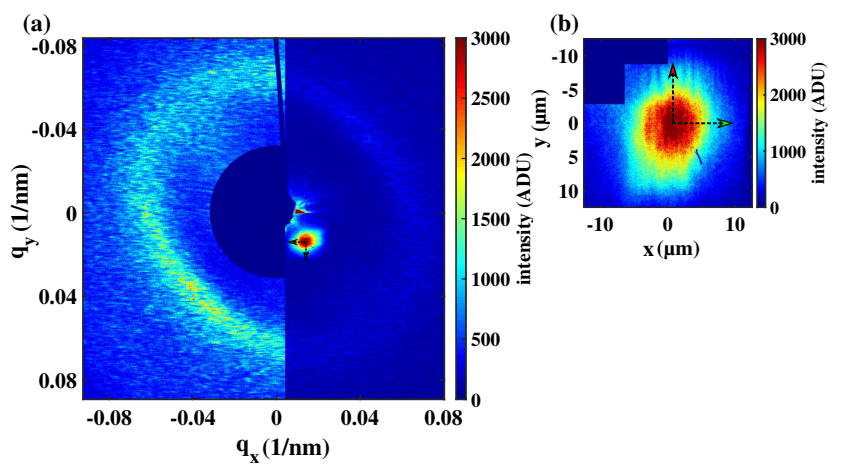

Fig. 2. (a) SAXS pattern of the partially coherent illuminated nanodot array together with the scattering signature of the curved grating. In the left half, the central part is masked out. The right half is shown with seven times reduced contrast to show the scattering signature of the curved grating, which is masked out on the left. (b) Beam intensity distribution extracted from (a) in real-space coordinates and correct orientation.
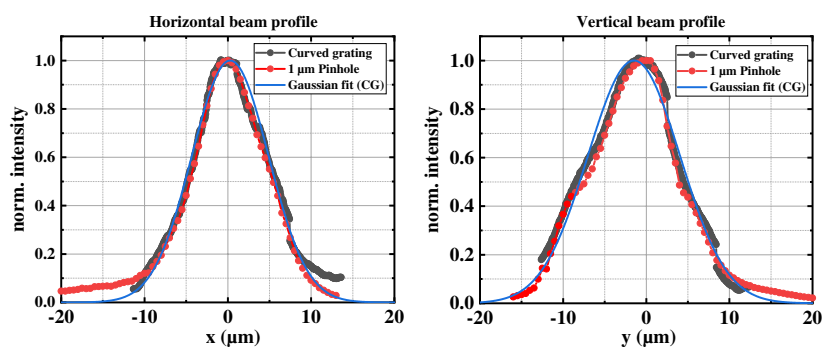

Fig. 3. Horizontal and vertical line profiles of the photon-beam intensity distribution obtained from the scattering signature of the curved grating (CG) (gray dots) and from scans using a $1 \mu \mathrm{m}$ pinhole (red dots). The blue lines are Gaussian fits to the curved-grating data.
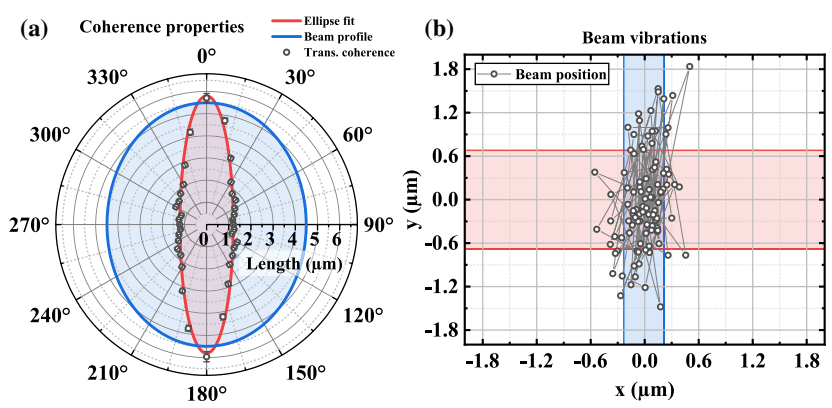

Fig. 4. (a) $2 \mathrm{D}$ transverse-coherence length (gray circles) and an ellipse fit to the data (red line). The blue line shows the rms widths of the beam-intensity distribution obtained from a 2D Gaussian fit to the 2D curved-grating data. (b) Horizontal $(x)$ and vertical $(y)$ photon-beam positions (gray circles) obtained from 2D Gaussian fits to $2 \mathrm{D}$ photon-beam intensity distributions. The blue- and red-shaded areas represent the standard deviations of the data in horizontal and vertical directions, respectively, being only a fraction of the beam size.

To validate the performance of the grating, the extracted photon-beam profile is compared to one measured scanning the beam with a $1 \mu \mathrm{m}$ pinhole on a photodiode downstream of the sample (see Fig. 3). The horizontal and vertical line profiles of the 2D photon-beam profile shown in Fig. 2(b) are extracted by averaging over $1 \mu \mathrm{m}$ in width across the beam center. The line profiles using the $1 \mu \mathrm{m}$ pinhole (red lines) are in very good agreement with the ones obtained from the $2 \mathrm{D}$ photon-beam profile [see Fig. 2(b)] (gray lines). A Gaussian fit to the latter profiles results in a beam width of $11 \mu \mathrm{m}$ and $13 \mu \mathrm{m}$ (FWHM) in horizontal and vertical directions, respectively.

The $2 \mathrm{D}$ spatial-coherence properties of the illuminating beam can be determined using the $2 \mathrm{D}$ photon-beam profile [see Fig. 2(b)], Eq. (3), and the procedure presented in Ref. [9]. Figure 4(a) shows the extracted transverse coherence lengths in all angular directions using the Fourier-analysis method and hence the spatial-coherence area of the illuminating beam. Using the obtained transverse coherence lengths in horizontal and vertical directions $l_{s, x}=(1.18 \pm 0.04) \mu \mathrm{m}$ and $l_{s, y}=(5.95 \pm 0.53) \mu \mathrm{m}$, respectively, global degrees of coherence of $\zeta_{x}=(13 \pm 1) \%$ and $\zeta_{y}=(50 \pm 3) \%$ are determined. This results in a total degree of coherence of $\zeta_{t}=\zeta_{x} \zeta_{y}=(6.5 \pm 2) \%$, which reflects mainly the large horizontal source size of PETRA III when using the full beam [10]. 
Vibrations of the photon beam caused by beamline optics vibrations could cause a spatial-coherence degradation, e.g., due to a smearing of the speckles within the SAXS pattern. This applies not only to the Fourier-analysis method, but also to typical X-ray imaging experiments. In order to probe coherence-degradation effects, time-resolved spatial-coherence measurements are required. In the here-presented experiments, the spatial-coherence properties are determined within a time frame of $500 \mathrm{~ms}$. This lower limit is set by the sensitivity of the detector and the scattering efficiency of the sample at the chosen photon energy. Below $500 \mathrm{~ms}$ exposure time, the scattered intensity of the sample is insufficient for the spatial-coherence determination. However, other diagnostic samples can be easily tailored in order to circumvent this limitation. However, direct observation of the photon-beam's spatial jitter in the sample plane is possible on shorter time scales owing to the stronger diffraction signal from the curved grating. We separately record 100 diffraction images with exposure times of $5 \mathrm{~ms}$ and obtain the center positions of the beam profiles by 2D Gaussian fits [see Fig. 4(b)]. Due to the detector read-out time of $4 \mathrm{~s}$, this yields only the the amplitude of the spatial jitter, but not the corresponding frequency. The analysis of the amplitudes results in a standard deviation of the beam movement of $200 \mathrm{~nm}$ in the horizontal and $680 \mathrm{~nm}$ in the vertical directions (one pixel $\hat{=} 53 \mathrm{~nm}$ ). This result is in very good agreement with a beamvibration analysis performed using a beam-diagnostic tool specifically designed to map out the full photon-beam caustic. The beam-vibration analysis using this tool revealed vertical beam-vibration amplitudes of around $125 \mathrm{~nm}$ in the horizontal and $500 \mathrm{~nm}$ in the vertical directions [10]. From simulated SAXS experiments using the disordered nanodots, we found that vibrations of $680 \mathrm{~nm}$ do not have any effect on the extracted spatial-coherence properties using the Fourier-analysis method. Hence, indirect spatial-coherence degradation is not present in the here-presented experiments.

In conclusion, we have demonstrated a direct 2D spatialcoherence measurement at the P04 beamline at PETRA III using the Fourier-analysis method in conjunction with a curvedgrating beam monitor. We have shown that this combination is an ideal photon-beam diagnostics tool for a direct and simple 2D spatial-coherence determination. The photon-beam profile illuminating the sample and the SAXS pattern of the sample are recorded simultaneously on the same detector exposure so that the only limiting time factor for the coherence analysis is the exposure time, which is $500 \mathrm{~ms}$ in the presented case. Further improvements to the nanodot sample in terms of material, thickness, and spatial distance distribution allow for coherence measurements on single-digit millisecond time scales as well as at tender X-ray photon energies due to the resulting higher scattering efficiency. Due to the particularly simple data analysis, the computational effort is low. Hence, the presented method can be used for a time-resolved spatial-coherence analysis as well as shot-to-shot-based measurements at free-electron laser sources, especially when high-frame-rate soft X-ray detectors become available. Particularly at fourth-generation synchrotron sources [17] offering extremely high spatial coherence, this method will help to identify possible sources of spatial-coherence degradation. As the Fourier-analysis method can be easily carried out in standard CDI, FTH, or XPCS experimental setups, it can be used for a quick 2D spatial-coherence determination and as a photon-beam optimization tool prior to or during X-ray scattering and imaging experiments.

Funding. Bundesministerium für Bildung und Forschung (FSP 301/05K10GU4); Deutsche Forschungsgemeinschaft (EXC 1074, EXC 2056, SFB 668, SFB 925).

Acknowledgment. We acknowledge DESY (Hamburg, Germany), a member of the Helmholtz Association HGF, for the provision of experimental facilities. The research was carried out at the P04 beamline at PETRA III. We thank Frank Scholz, Jörn Seltmann, and Jens Buck for support during the experiments.

Disclosures. The authors declare no conflicts of interest.

\section{REFERENCES}

1. Y. Wang, T. Xiao, and H. Xu, J. Synchrotron Rad. 7, 209 (2000).

2. S. Goto, Proc. SPIE 9588, 95880G (2015).

3. J. Miao, P. Charalambous, J. Kirz, and D. Sayre, Nature 400, 342 (1999).

4. S. Eisebitt, J. Lüning, W. F. Schlotter, M. Lörgen, O. Hellwig, W. Eberhardt, and J. Stöhr, Nature 432, 885 (2004).

5. D. Stickler, R. Frömter, H. Stillrich, C. Menk, C. Tieg, S. StreitNierobisch, M. Sprung, C. Gutt, L.-M. Stadler, O. Leupold, G. Grübel, and H. P. Oepen, Appl. Phys. Lett. 96, 042501 (2010).

6. D. L. Abernathy, G. Grübel, S. Brauer, I. McNulty, G. B. Stephenson, S. G. J. Mochrie, A. R. Sandy, N. Mulders, and M. Sutton, J. Synchrotron Rad. 5, 37 (1998).

7. M. D. Alaimo, M. A. C. Potenza, M. Manfredda, G. Geloni, M. Sztucki, T. Narayanan, and M. Giglio, Phys. Rev. Lett. 103, 194805 (2009).

8. X. Shi, S. Marathe, M. J. Wojcik, N. G. Kujala, A. T. Macrander, and L. Assoufid, Appl. Phys. Lett. 105, 041116 (2014).

9. K. Bagschik, R. Frömter, L. Müller, W. Roseker, J. Bach, P. Staeck, C. Thönnißen, S. Schleitzer, M. H. Berntsen, C. Weier, R. Adam, J. Viefhaus, C. M. Schneider, G. Grübel, and H. P. Oepen, Opt. Express 24, 23162 (2016).

10. K. Bagschik, J. Wagner, R. Buß, M. Riepp, A. Philippi-Kobs, L. Müller, J. Buck, F. Trinter, F. Scholz, J. Seltmann, M. Hoesch, J. Viefhaus, G. Grübel, H. P. Oepen, and R. Frömter, Opt. Express 28, 7282 (2020).

11. M. Schneider, C. M. Günther, C. von Korff Schmising, B. Pfau, and S. Eisebitt, Opt. Express 24, 13091 (2016).

12. M. Schneider, C. M. Günther, B. Pfau, F. Capotondi, M. Manfredda, M. Zangrando, N. Mahne, L. Raimondi, E. Pedersoli, D. Naumenko, and S. Eisebitt, Nat. Commun. 9, 214 (2018).

13. J. Viefhaus, F. Scholz, S. Deinert, L. Glaser, M. Ilchen, J. Seltmann, P. Walter, and F. Siewert, Nucl. Instrum. Methods Phys. Res. A 710, 151 (2013).

14. A. Neumann, N. Franz, G. Hoffmann, A. Meyer, and H. P. Oepen, Open Surf. Sci. J. 4, 55 (2012).

15. I. A. Vartanyants and I. K. Robinson, J. Synchrotron Rad. 10, 409 (2003).

16. L. Mandel and E. Wolf, Optical Coherence and Quantum Optics (Cambridge University, 1995).

17. C. G. Schroer, I. Agapov, W. Brefeld, R. Brinkmann, Y.-C. Chae, H.C. Chao, M. Eriksson, J. Keil, X. Nuel Gavalda, R. Röhlsberger, O. H. Seeck, M. Sprung, M. Tischer, R. Wanzenberg, and E. Weckert, J. Synchrotron Rad. 25, 1277 (2018). 